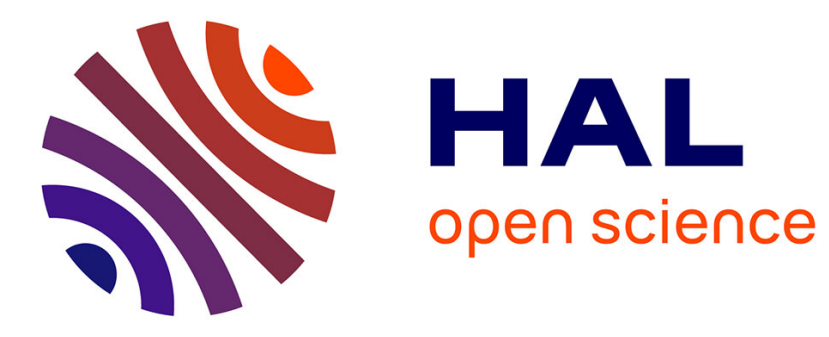

\title{
Learning-based thermal rendering in telepresence
}

\author{
Mohamed Guiatni, Abdelaziz Benallegue, Abderrahmane Kheddar
}

\section{To cite this version:}

Mohamed Guiatni, Abdelaziz Benallegue, Abderrahmane Kheddar. Learning-based thermal rendering in telepresence. EuroHaptics, Jun 2008, Madrid, Spain. , 6th International Conference on Human Haptic Sensing and Touch Enabled Computer Applications, LNCS (5024), pp.820-825, 2008, Haptics: Perception, Devices and Scenarios. 10.1007/978-3-540-69057-3_103 . lirmm-00798803

\section{HAL Id: lirmm-00798803 https://hal-lirmm.ccsd.cnrs.fr/lirmm-00798803}

Submitted on 25 Sep 2019

HAL is a multi-disciplinary open access archive for the deposit and dissemination of scientific research documents, whether they are published or not. The documents may come from teaching and research institutions in France or abroad, or from public or private research centers.
L'archive ouverte pluridisciplinaire HAL, est destinée au dépôt et à la diffusion de documents scientifiques de niveau recherche, publiés ou non, émanant des établissements d'enseignement et de recherche français ou étrangers, des laboratoires publics ou privés. 


\title{
Learning-Based Thermal Rendering in Telepresence
}

\author{
Mohamed Guiatni ${ }^{1}$, Abdelaziz Benallegue ${ }^{2}$, and Abderrahmane Kheddar ${ }^{3}$ \\ ${ }^{1}$ Military Polytechnical School, Algiers, Algeria \\ ${ }^{2}$ LISV, University of Versailles, France \\ ${ }^{3}$ Centre National de la Recherche Scientifique, France \\ guiatni@iup.univ-evry.fr, benalleg@lisv.uvsq.fr, kheddar@ieee.org
}

\begin{abstract}
We propose a new method for thermal rendering in telepresence systems which allows an operator to feel most transparently the thermal behavior of the remote object. It is based on learning and thermal heat flux generation. Two databases are constructed from real measurements recorded during direct contact between operator's finger and different materials. One database is used in order to identify the material in the slave side, the other database is used to generate desired heat flux for the thermal display loop. The identification bloc is based on Principal Component Analysis and Neural Network. Experimental results validating the proposed method are discussed.
\end{abstract}

Keywords: Thermal display, Heat flux control, Learning, Telepresence.

\section{Introduction}

There has been several work in thermal sensing and rendering, see e.g. 6] [2] 7] [3] [1] [5. In this paper, we address thermal rendering in real telepresence. To overcome the lack of having a remote sensing system with exact replication of the human fingertip, we propose a new approach that shall improve thermal sensation and produce more realistic thermal feedback when a finger touches a remote object. For a given thermal sensing technology, when the contact occurs in the remote part, the material is identified first based on our method, then adequate heat flux is generated to be used as desired flux for the thermal display in the operator side. To realize material identification, we proceed to experimental observations of the heat exchange which occurs when a fingertip touches several objects having different thermal parameters; these objects are used as standard samples. These observations are used to build a database by recording the heat flux and temperature evolution for each material. Another database results from the observation of contact between the material samples and the slave device.

The observation database is used to train a classifier. When the material is identified in the telepresence site, an identifier built-in controller provide adequate desired signals for both the sensor and the display. An additional sensor is also used in order to detect binary contact states and the force applied by the operator. 


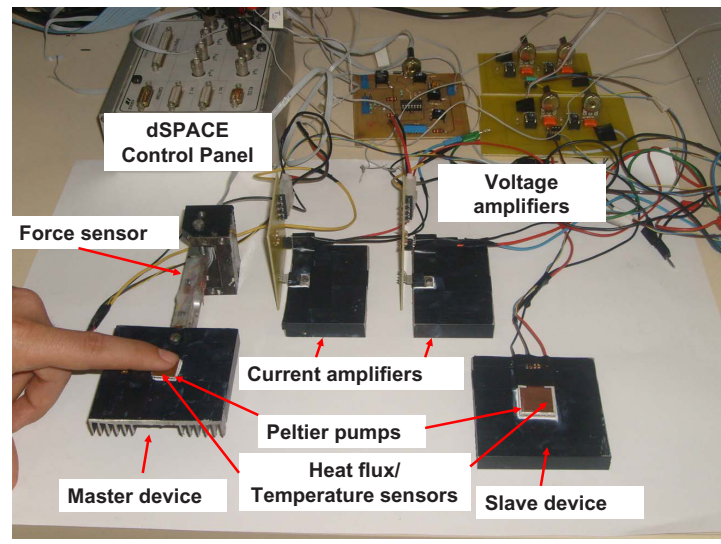

Fig. 1. Master/slave thermal teleoperator: the left part is the display and the right part, identical to the left system, is used as a thermal sensor

\section{Experimental Setup}

Figure 1 shows our experimental setup of the master/slave thermal teleoperator. Each part consists of a contact pad using a Peltier heat pump of dimension $15 \times 15 \mathrm{~mm}$ with a thickness of $400 \mu \mathrm{m}$ from MELCOR Corporation. A heat flux sensor with an integrated thermocouple type $\mathrm{T}$ from Captec is assembled at the contact interface with the Peltier pump to measure simultaneously both the temperature and the heat flux, the sensor sensibility is about $1.26 \mu \mathrm{V} /\left(\mathrm{W} / \mathrm{m}^{2}\right)$. The underside of the Peltier pump is placed on a dissipater in order to keep its temperature constant. A silicone grease is used to decrease the contact resistance at the contact surface of the Peltier pump with the temperature/heat flux sensor. In the master side, a force sensor is introduced in order to detect the contact and measure the force applied by the operator finger. In the slave side a pressure sensor is also incorporated in order to detect eventually contact between the slave pad and objects. A radiation thermometer is used for measuring the temperature of the room, the object and the operator finger before contact. Supervision and control are performed by means of a dSPACE DS1103 setup. Data are sampled at a frequency of $100 \mathrm{~Hz}$.

\section{Material Identification and Heat Flux Generation}

Two databases are constructed: the first is used to train and to validate the material identification bloc. The second allows the generation of the adequate signal to be tracked at contact. Seven different materials are selected. These materials are characterized by different thermal properties and equal volume. For constructing a database for thermal interaction, we proceeded to make ten tests with each material. At first, the room temperature is measured using infrared 
thermometer, which gives us the initial temperature of the material. Then, the Peltier device is heated until the temperature be as $10^{\circ} \mathrm{C}$ greater than that of the samples taken as reference (figures 2) (Which corresponds to the average of the initial gap between the temperature of the finger and that of the samples). For each experiment, heat flux signal is recorded and filtered beginning from the first instance of contact between the pad and the material.

\subsection{PCA Algorithm}

Principal Component Analysis is used in order to reduce the data dimension and extract useful information. First, the average signal is computed as:

$$
x_{m}(t)=\frac{1}{N} \sum_{i=1}^{N} x_{i}(t) \quad t=0, \ldots, M
$$

where $x_{i}$ is the recorded data for a sample $i, N$ the size (cardinality) of the learning set and $M$ the length of the sampled data. Then, the average is subtracted from the original data, i.e. $y_{i}(t)=x_{i}(t)-x_{m}(t)$ and the data is prearranged in a matrix format:

$$
Y_{(M+1) \times N}(t)=\left[y_{1}(t), \ldots, y_{i}(t), \ldots, y_{N}(t)\right] ; t=0, \ldots, M
$$

Let $A=\frac{1}{\sqrt{N}} Y^{T}$, we get the new orthogonal base as follow:

$$
\phi_{i}=\frac{1}{\sqrt{\lambda_{i}}} A \nu_{i}^{T}
$$

where, $\lambda_{i}$ is the eigenvalue $i$, and $\nu_{i}$ is the corresponding eigenvector of the covariance matrix $C=A A^{T}$. For each measurement, the projection of $Y$ on the base $\Phi$, gives $u_{i}=y_{i}^{T} \Phi, u_{i}$ is then the new representation of the sample $i$.

\subsection{FNN Algorithm}

Feedforward Neural Network (FNN) with one hidden layer is used. The number of neurons in the input layer is chosen to be the dimension of the input vector, and the number of neurons of the output layer is equal to the number of classes (seven). Sigmoid activation functions are used. The following criterion determines the size of the error to be back propagated. The mean squared error criterion is used:

$$
E=\frac{1}{2} \frac{1}{N} \sum_{k=1}^{N} \sum_{p=1}^{N_{o}}\left[d_{k}^{p}-z_{k}^{p}\right]^{2}
$$

where $d_{k}^{p}$ is the desired output for unit $k$ when pattern $p$ is clamped, $z_{k}^{p}$ the actual output and $N_{o}$ the number of output. The objective during training is to minimize this error by choosing the appropriate weights. In this paper we use the well-known Levenberg-Marquardt rule: $H_{i j}=\frac{\partial^{2} E}{\partial \omega_{i j} \partial \omega_{j k}}$, where $H$ is the Hessien matrix. The new weight value is determined by the learning algorithm: 

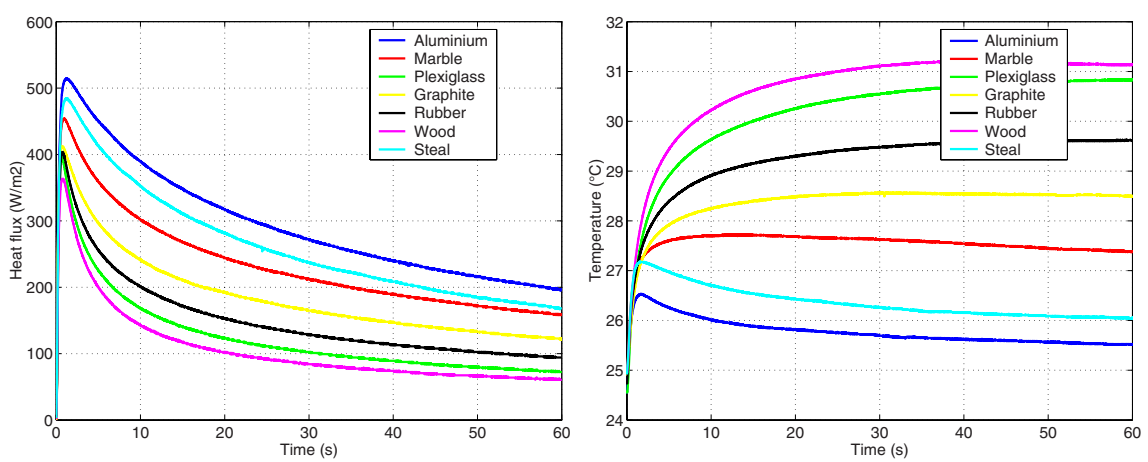

Fig. 2. Left: Thermal heat flux exchanged during real contact between finger and materials, Right:Temperature evolution during real contact between finger and materials.

$$
\omega_{t+1}=\omega_{t}-(H+\lambda I)^{-1} \Lambda^{T} \operatorname{err}\left(\omega_{t}\right)
$$

where $\Lambda_{i}=\frac{\partial \boldsymbol{x}_{k}}{\partial \omega_{i}}$ and $\operatorname{err}\left(\omega_{t}\right)$ is the error on the previous weights.

Experiments were performed with five persons. Before each experiment, the temperatures of the index finger of the subject and the material are measured. Subjects were instructed to keep their index finger on the support and to apply a force of $\approx 10 \mathrm{~N}$ of course we instruct the user not to focus on this issue; we wanted to get a similar contact surface in most cases. The exchanged heat flux is recorded during 60 seconds, a sample profile is presented in figure 2. The difference between the heat flux profiles and the temperature profiles is apparent. In order to get normalized data, we divide each measurement by the initial temperature gap between the operator finger and the material that is to be touched. According to the length of the presented data, the performance of the identification bloc is affected. For a 1sec sequence length, and seven classes, we have a confusion in one hand, between aluminum and steel and in the other hand, between graphite and rubber. This is probably due to their close thermal properties. By gathering the confused materials in two classes, the result is improved though for sequences of $0.75 \mathrm{sec}$ ( $100 \%$ of good identification). But if we decrease more the sequences length, the performance will be degraded, (table 1).

For the second database, for each material, the average data was computed and used to train a feed-forward neural networks bloc responsible of the generation of desired flux for to be displayed to the user. We used one neural network with linear output for each material and the same algorithm presented previously.

\section{Experiments}

During all the experiments, a PID controller is used in the heat flux control loop. Contact is established at the same time both in the master and the slave 
Table 1. Validation results with five classes (columns) $(\oplus$ classification with $0.25 \mathrm{sec}$ sequence length, $\otimes$ classification with $0.5 \mathrm{sec}$ sequence length)

\begin{tabular}{|c|c|c|c|c|c|}
\hline & Aluminium-Steel & Marbre & Plexiglass & Graphite-Rubber & Wood \\
\hline Aluminium & $\begin{array}{l}\oplus \oplus \oplus \oplus \oplus \\
\otimes \otimes \otimes \otimes \otimes\end{array}$ & & & & \\
\hline Marble & $\begin{array}{c}\oplus \oplus \\
\otimes\end{array}$ & $\begin{array}{c}\oplus \oplus \oplus \\
\otimes \otimes \otimes \otimes\end{array}$ & & & \\
\hline Plexiglass & & & $\begin{array}{c}\oplus \oplus \oplus \\
\otimes \otimes \otimes \otimes \otimes\end{array}$ & $\oplus$ & $\oplus$ \\
\hline Graphite & & & & $\begin{array}{c}\oplus \oplus \oplus \oplus \\
\otimes \otimes \otimes \otimes \otimes\end{array}$ & $\oplus$ \\
\hline Rubber & & $\oplus$ & & $\begin{array}{c}\oplus \oplus \oplus \oplus \\
\otimes \otimes \otimes \otimes \otimes\end{array}$ & \\
\hline Wood & & & & & $\begin{array}{l}\oplus \oplus \oplus \oplus \oplus \\
\otimes \otimes \otimes \otimes \otimes\end{array}$ \\
\hline Steel & $\begin{array}{l}\oplus \oplus \oplus \oplus \oplus \\
\otimes \otimes \otimes \otimes \otimes\end{array}$ & & & & \\
\hline
\end{tabular}
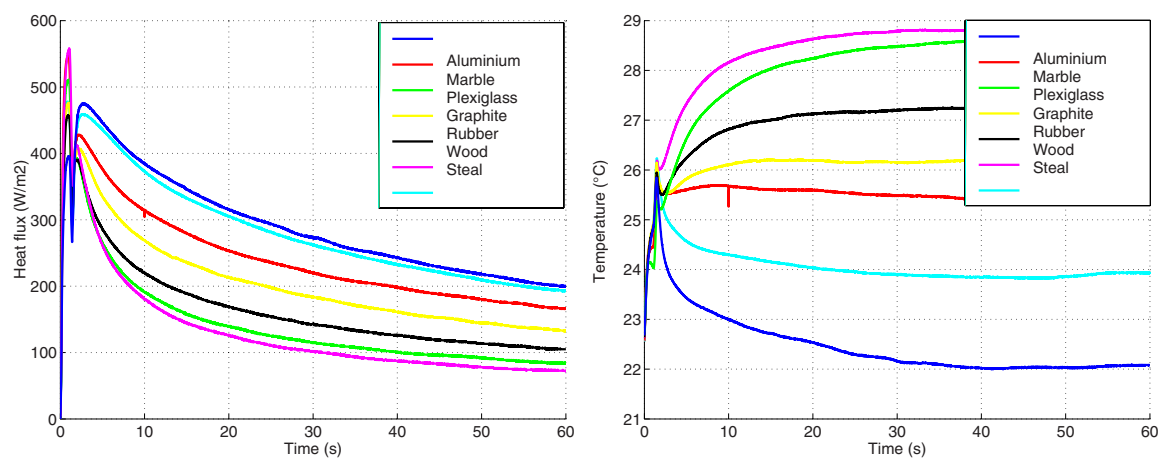

Fig. 3. Left: Exchanged heat flux, Right: Temperature evolution during contact in the master side

side. The resulting desired flux is sent to the master and the slave heat flux control loops. During the first instant of contact, the heat flux exchanged is due to the contact between the operator finger and the master pad. So that (figure [3), the first thermal exchange is driven by intrinsic properties of the master pad because of the latency required for material identification. Although no temperature control is performed, the temperature profiles are very similar to those recorded during direct contact. This scenario is similar to a situation where a non-wearable haptic device is used for teleoperation. In all cases, the heat flux is reproduced accurately. The temperature profiles are similar to those recorded during direct contact. This result shows that we have an interaction very similar to that observed during direct contact. 


\section{Conclusion}

To reproduce realistic thermal sensations, we propose a new approach based on a learning technique using real measurements of thermal heat flux exchanged during contact between operator finger and several materials. The candidates' materials are chosen so that they have dissimilar thermal properties. The identification of the material allows the generation of the adequate desired heat flux. The percentage of good classification depends on the length of the temporal sequence used for learning and/or for validation and the performance of the rendering depends on the instant and the type of contact. To validate this approach, an experimental setup is realized and a set of experiment is performed. The obtained results are encouraging and allow the improvement of the thermal rendering in the future work in order to get more realistic sensations when exploring or manipulating remote objects via haptic interfaces.

Acknowledgments. This work is partially supported by grants from the ImmerSence EU CEC project, Contract No. 27141 www.immersence.info/ (FETPresence) under FP6. The authors would like to thank M. Lehtihet.

\section{References}

1. Benali-Khoudja, M., Hafez, M., Alexandre, J.-M., Benachour, J., Kheddar, A.: Thermal feedback model for virtual reality. In: IEEE International symposium on Micromechatronics and Human Science (October 2003)

2. Caldwell, D.G., Andersen, U., Bowler, C., Wardle, A.: A high power/weight dexterous manipulator using sensory glove based motion control and tactile feedback. Transaction of the Institute of Measurement and Control 17(5), 234-241 (1995)

3. Drif, A., Citérin, J., Kheddar, A.: Thermal bilateral coupling in teleoperators. In: IEEE/RSJ International Conference on Robots and Intelligent Systems, Canada, August 2-6 (2005)

4. Ho, H., Jones, L.: Material identification using real and simulated thermal cues. In: 26th Annual International Conference of the IEEE EMBS, pp. 2462-2465 (September 2004)

5. Jones, L.A., Berris, M.: The psychophysics of temperature perception and thermalinterface designs. In: IEEE International symposium on Micromechatronics and Human Science (2002)

6. Russell, R.A.: Thermal sensor for object shape and material constitution. Robotica 6, 31-34 (1988)

7. Yamamoto, A., Cros, B., Hashimoto, H., Himchi, T.: Control of thermal tactile display based on prediction of contact temperature. In: IEEE International Conference on Robotic and Automation, pp. 1536-1541 (April 2004) 